\title{
Role of radiologically guided FNAC in determining the lesions of spine
}

\author{
Shukla $A^{1}$, Iyenger $S^{2}$, Jasmeet ${ }^{3}$, Iyenger $S . N^{4}$ \\ ${ }^{1}$ Dr Avdhesh Shukla, Assistant professor, Department of Neurosurgery, ${ }^{2}$ Dr Sudha Iyenger, Associate professor \\ Department of pathology, ${ }^{3}$ Dr Jasmeet, Resident, Department of Neurosurgery, ${ }^{4}$ Dr SN Iyenger, Professor, Department \\ of Neurosurgery. All are affiliated with G.R.M.C, Gwalior, MP, India
}

Address for correspondence: Dr Avdhesh Shukla, Email: roshanchanchlani@gmail.com

\begin{abstract}
Introduction: The vertebral column is an important site for occurrence of different variety of inflammatory, benign and malignant lesions. The advantages of the image guide FNAC are: Minimal invasive, relatively inexpensive, accurate, outdoor procedure, minimal complication, takes shorter time for diagnosis and can be repeated. Objective: find out relation between diagnosis made by radiological guided FNAC and clinoco-radiological findings. Methodology: The present cross-sectional hospital based study was carried out in the Department of Neurosurgery, G.R.M.C., Gwalior from Nov. 2011 to March 2014. Patients of age 5 yrs and above with neurological signs and symptoms due to spinal lesions having normal coagulation profile and consenting to participate were included in the study. Each under went clinicradiological examination and radiologically guided FNAC. Results: All the patients who were clinic-radiologically suspected as case of Pott's spine were confirmed on FNAC as Pott's spine (35 out of 35). The patients in whom metastasis was presumptive diagnosis, on FNAC only 9 out of 21 i.e. $42.86 \%$ turned out to have metastases of spine, rest of the patients were found to have Pott's spine (7 i.e. 33.33\%), multiple myeloma (4 i.e. 19.05\%) on cytological confirmation. Conclusion: Patients with Pott's spine should undergo radiologically guided FNAC as a routine part of their work up to rule out metastasis of the spine.
\end{abstract}

Keywords: Fine Needle Aspiration Cytology, Potts spine, Spine, Tumours.

\section{Introduction}

The retroperitoneum and the spine have long been areas of curiosity and interest with regard to the diagnosis of lesions occurring in these regions, more so because the usual diagnostic armamentarium falls short of providing the requisite access [1]. The vertebral column is an important site for occurrence of different variety of inflammatory, benign and malignant lesions. Since the common presenting complaint of all these lesions is pain, it becomes important to diagnose these lesions by using clinical acumen and best available diagnostic modalities. However, considering numerous and heterogeneous contents of these regions, lesions that can be encountered potentially are numerous and usual diagnostic armamentarium falls short of providing the exact diagnosis of the lesion. FNAC of radiologically detected vertebral and paravertebral lesions is now exclusively used for diagnosis and further management. It can be safely performed at difficult sites, does not

Manuscript received: $1^{\text {st }}$ Aug 2015

Reviewed: $7^{\text {th }}$ Aug 2015

Author Corrected: $17^{\text {th }}$ Aug 2015

Accepted for Publication: $26^{\text {th }}$ Aug 2015 require hospitalization, allows preliminary diagnosis in 15-20 min of aspiration and adjuvant methods such as electron microscopy, immunocytochemistry and DNA

ploidy can be employed to arrive at definitive diagnosis [2].

This (FNAC) is of particular importance in suspected infection where a chemotherapeutic agent may be indicated and in suspected metastatic disease where it may be helpful in identifying the nature and probable site of the primary tumour. Moreover, a solitary vertebral lesion in a patient with known malignant disease does not always indicate metastasis. The most common pathological diagnosis is tuberculosis of the vertebral column (Pott's spine) [3,4]. There are on an average 30 million gross tuberculosis patients globally and approximately one third of the cases are found in India. 1 to $3 \%$ of the 10 million have involvement of bone and joints. Vertebral tuberculosis is the commonest form of skeletal tuberculosis comprising about $50-70 \%$ of all bone and joint tuberculosis [5]. It 
may present as bony vertebral pain or as paraparesis. Tissue diagnosis is required before initiation of therapy, as sometimes it is not possible to differentiate inflammatory lesions from a malignancy. Thus the role of FNAC is of utmost relevance in this setting as it avoids unnecessary open biopsy in these morbid patients [6].

The vertebral column is a common site of metastasis. FNAC is associated with a lower risk of tumour seeding as compared to open biopsy. One of the most important indications for FNAC of a radiologically detected vertebral lesion in a patient with a prior history of malignancy is to establish the presence of metastasis. In patients with no known primary tumour, FNAC can be helpful in establishing an initial diagnosis of malignancy and initiate a search for the occult primary malignancy. The common primary sites with vertebral metastasis are lung and breast $[3,4]$.

The first CT guided FNAC of spine was done by Adapon (1981) with 50\% accuracy rate. But with advances in CT technology as well as in the cytology techniques, percutaneous aspiration cytology (PAC) rather than the 'core biopsy' has gained momentum [7]. The advantages of the image guide FNAC are: Minimally invasive, relatively inexpensive, accurate, outdoor procedure, minimal complication takes shorter time for diagnosis and can be repeated. This project was undertaken to find out relation between diagnosis made by radiological guided FNAC and clinic-radiological findings.

\section{Material and Methods}

The present cross-sectional hospital based study was carried out in the Department of Neurosurgery, G.R.M.C, Gwalior from Nov. 2011 to March 2014. After the institutional ethics committee permission was sought, all the patients of age 5 years and above from either sex presented to the Neurosurgical O.P.D. with neurological signs and symptoms due to spinal lesions were evaluated and investigated and only those with confirmed solitary/multiple vertebral lesions on $\mathrm{x}$ ray/CT/MRI, having normal coagulation profileand consenting to participate were included in the study. The pre-procedure work up of these patients was as follows: Detailed clinical evaluation (history and physical examination). Laboratory studies which include: Complete hemogram, complete coagulation profile i.e. bleeding time, coagulation time, prothrombin time, prothombin time index and platelet count and
Urine examination. Radiological studies included Spinal radiogarph, antero-posterior and lateral views;

Chest radiograph - PA view; MRI of spine/CT of spine and Ultrasound abdomen and pelvis when indicated. Percutaneous spine FNAC was performed with local anesthesia or local anesthesia and conscious sedation. To minimize the possibility of infection, the study was performed with strict aseptic technique.

Patient positioning depends upon the spine level (cervical, thoracic, or lumbosacral) of the lesion and its location (vertebral body versus posterior elements). The prone position is optimal for accessing lesions in the thoracic or lumbosacral spine or, rarely, within the posterior aspect of the cervical spine. The supine position is usually required to access the cervical spine. In certain instances - for example, when a patient cannot lie completely prone - the lateral decubitus or prone oblique position can be helpful, an intravenous catheter was also placed prior to the procedure to facilitate the intravenous administration of medications, contrast agents, or hydration.

Equipment requirements: Image guidance was accomplished with several different modalities. These included fluoroscopy, computed tomography, computed tomography combined with a multidirectional fluoroscope, computed tomographic fluoroscopy, and magnetic resonance imaging. The choice of equipment was determined by its availability, operator preference, and by the location and size of the suspected lesion. A CT-guided spine biopsy was performed without or with the use of a stereotactic apparatus to guide the insertion of the biopsy needle. The use of MRI required the simultaneous usage of MR-compatible equipment, both for patient monitoring and for performing the biopsy procedure.

Biopsy Techniques: An important decision that was made before and during spine FNAC is the choice of approach. The determinants for the approach were lesion location and lesion size. A posterior approach is used for thoracic, lumbosacral, and posterior cervical lesions. An anterior approach was used for most cervical spine biopsies. The location of "critical" normal anatomical structures also modified the approach. Unless the lesion was clearly localized to the left side of the spine, a right-sided approach was preferred to a left-sided approach for accessing thoracic spine tumors without damaging the aorta. The objective was to choose a trajectory that enables access to the 
lesion without compromising normal, critical structures. The specific location of the lesion within the spine also influenced the approach that was selected. A vertebral body lesion and a posterior element lesion were approached differently.The selected imaging modality was used to identify the lesion level. Once a safe path to the target lesion was chosen, the entry site on the skin surface was marked with an indelible ink marker. The region of interest was prepared and draped in sterile fashion. A $1 \mathrm{~cm}$ wheal was raised at the skin entry site by using a 25 -gauge needle and a local anesthetic agent (e.g., $1 \%$ lidocaine, $0.25 \%$ bupivacaine). A styletbearing thin needle was then advanced by means of image guidance, and the local anesthetic was administered into the deeper soft tissues. If a vertebra was to be entered, infiltration of the anesthetic agent into the periosteum was extremely helpful in minimizing patient discomfort. When the needle tip was in satisfactory position, the needle hub was removed. Specimen retrieval by means of fine-needle aspiration requires an in-and-out motion within the lesion matrix. To access bone marrow or a lytic lesion with an aspiration or cutting needle, a pre-existing bone window must be present within the vertebral cortex, as occurs with a lytic focus, or a cortical window was first to be cut with a bone needle. Neither aspiration nor cutting needles will penetrate normal or near normal bone cortex. The suction was released and in this position, both the needle and the syringe were withdrawn. It is important to release negative pressure before removing the needle and syringe and if this is forgotten then the material from the needle may be accidentally aspirated into the syringe and it becomes difficult to expel it. Needle is detached from syringe and material expelled onto glass slides and thin smears are prepared. If infection was suspected clinically and radiologically, part of the aspirate was sent to the microbiology department for routine bacterial stains as well as A.F.B. culture. Post procedure $\mathrm{x}$-ray chest in erect posture is performed in case of thoracic spine FNAC to exclude pneumothorax.Analgesics were given if patient complains of pain. Any complaints e.g. chest pain, neurological deficit etc are recorded and managed accordingly.

\section{Results}

A total of 56 cases were included in this study. The age of patients ranges between 13 to 72 yrs and there be 19 females $(33.93 \%)$ and 37 males (66.07\%). Maximum cases were in 3rd to 5th decades. The mean age of cases was $43.2 \pm 12.3$ years. In males the mean age (46.3 \pm 11.1 years) was higher than females $(40.1 \pm 14.2$ years). Pain was the most common presenting symptom, followed by radiculopathy and weakness which were seen in $35(62.5 \%)$ and 15 patients (26.79\%) respectively. Sphincter disturbances were seen in 6 patients (10.71\%) (Table No. 1). Lumbar spine (31 i.e. 55.35\%) was most commonly involved segment followed by lower dorsal spine (13 i.e. 23.22\%). Upper dorsal and cervical spine was involved in 2 patients each while 7 patients had involvement of multiple spinal segments. L3-L5 segments showed involvement in almost one third of the cases (15 cases). Overall there was uniform distribution in lumbar segment with slight preponderance in the L4-L5 segment. Being a referral center many patients came here after being investigated at many places. Most of the patients level of exact lesion was decided on the basis of the MRI of the involved spine (Table No. 2). Many patients had signs and symptoms of systemic involvement (Table No.3). In maximum number of patients (50 i.e. 89.29) FNAC was done under CT guidance. All the patients who were clinic-radiologically suspected as case of Pott's spine were confirmed on FNAC as Pott's spine (35 out of 35). The patients in whom metastasis was presumptive diagnosis, on FNAC only 9 out of 21 i.e. $42.86 \%$ turned out to have metastases of spine, rest of the patients were found to have Pott's spine (7 i.e. 33.33\%), multiple myeloma (4 i.e. 19.05\%) on cytological confirmation (Table No.4).

Table No. 1: The clinical profile of the case

\begin{tabular}{|l|l|l|l|}
\hline S.No. & Clinical Features & No. & \% \\
\hline 1 & Pain & & \\
\hline & Neck & 2 & 3.57 \\
\hline & Upper backache & 10 & 17.86 \\
\hline & Low backache & 44 & 78.57 \\
\hline 2 & Radiculopathy & 35 & 62.5 \\
\hline 3 & Weakness & 15 & 26.79 \\
\hline 4 & Numbness & 12 & 21.43 \\
\hline 5 & Sphincter disturbance & 6 & 10.71 \\
\hline
\end{tabular}


Table No. 2: MRI findings of involved segments

\begin{tabular}{|l|l|l|l|}
\hline S.No. & MRI Finding & No. $(\mathbf{n}=\mathbf{5 6})$ & $\mathbf{\%}$ \\
\hline 1 & Predominant discal involvement & 7 & 12.5 \\
\hline 2 & Vertebral body and discal involvement & 6 & 10.71 \\
\hline 3 & Vertebral body destruction & 8 & 14.29 \\
\hline 4 & Vertebral body destruction with granulation tissue & 28 & 50 \\
\hline 5 & Abscess & 5 & 8.93 \\
\hline 6 & Reduce bone height without any other change & 15 & 26.78 \\
\hline
\end{tabular}

Table No. 3: Other systemic findings

\begin{tabular}{|l|l|l|l|}
\hline S.No. & Systemic findings & No. $(\mathbf{n}=\mathbf{5 6})$ & $\mathbf{\%}$ \\
\hline 1 & Abnormal chest x-ray & 10 & 45.46 \\
\hline 2 & USG abdomen pelvis (abnormal findings) & 4 & 18.18 \\
\hline 3 & CT abdomen & 0 & 0 \\
\hline 4 & Specific blood investigations: & & \\
\hline & PSA & 3 & 13.64 \\
\hline & Serum electrophoresis for M-Band & 4 & 18.18 \\
\hline & Hormonal profile e.g. (thyroid function test) & 1 & 4.54 \\
\hline & Others & 0 & 0 \\
\hline
\end{tabular}

Table No. 4: Comparison of clinic-radiological diagnosis with the radiological guided FNAC results

\begin{tabular}{|l|l|l|l|}
\hline S. No. & Findings & No. & $\%$ \\
\hline 1 & Clinicoradiological metastases & 21 & 100 \\
\hline 2 & radiologically guided FNAC results & 21 & 100 \\
\hline & Metastasis & 9 & 42.86 \\
\hline & Pott's & 7 & 33.33 \\
\hline & Multiple myeloma & 4 & 19.05 \\
\hline & Others & 1 & 4.76 \\
\hline
\end{tabular}

\section{Discussion}

The present study was done with an aim of to find out relation between diagnosis made by FNAC and radiological findings. The mean age of cases was $43.2 \pm$ 12.3 years. In males the mean age $(46.3 \pm 11.1$ years $)$ was higher than females $(40.1 \pm 14.2$ years), and there were fewer females than the males. Similar finding has been reported by Moller et al [8]. Our study observed pain as the most common presenting symptom followed by spinal deformity which are similar to findings by Moller et al[7]. As is clear from table above Dorsal and Lumbar spine was most commonly affected in our study (26.7\% and $55.35 \%$ respectively). This compares favourably with results of Mondal et al[9] and Moller et al[7]. Absence of cervical cases in the study of Moller et al [7] probably reflects patient selection.The most common MRI finding in the present study was vertebral body destruction with granulation tissue formation, which reflects the incidence of Pott's spine in this part of the world. Abscess formation was seen in $9 \%$ of the patients.A detailed workup all the patients was done to rule out source of spinal lesion. The chest $\mathrm{x}$-ray abnormality, which were most commonly observed were COPD changes and pleural effusion. USG of abdomen and pelvis which was done routinely did not show primary lesion/ concurrent second lesion in the patients who were included in the study. Serum PSA was raised in 3 patients. Spinal lesions in these patients turned out to be metastasis from carcinoma prostate. Mband electrophoresis was positive in 4 patients who were later diagnosed as cases of multiple myeloma on cytology report. In the present study Pott's spine was the most frequent spinal lesion encountered which reflects prevalence of Pott's spine in this part of world as well as poor socioeconomic status of the patients presenting to Govt. Hospitals. Paravertebral abscess 
was seen in $9 \%$ of the patients in present series but only in half of the patients AFB (Acid Fast Bacillus) was seen on routine microscopy. Metastasis of spine was seen in $15.79 \%$ of the patients and multiple myeloma contributed to $7 \%$ of spinal lesions. In various studies published world wide metastasis as well as infective osteomyelitis account for majority of spinal lesions [810]. Primary bone lesions other than multiple myeloma were not included in present study. The overall accuracy of various studies [8-10] range from 60-96\%. The accuracy of earlier studies is low probably because advances made in image guidance have helped to accurately localize the lesion as well as to guide placement of needle within the area of interest. In post CT era, the accuracy approaches $90-96 \%$. The accuracy of our study is $96.49 \%$ and this compares favorably with studies performed in post CT era. All the patients who were clinic-radiologically suspected as case of Pott's spine were confirmed on FNAC as Pott's spine. The patients in whom metastasis was presumptive diagnosis on clinico-radiologically, on FNAC only 9 out of 21 i.e. $42.86 \%$ turned out to have metastases of spine. Thus, radiologically guided FNAC is imported predictor in the diagnosis specially in the lesions of the spine.

\section{Conclusion}

Pain is the important symptom which brings the patients to the doctor. Mostly Lumber segment wasinvolved.CT guided FNAC is the most commonly used modality. All the patients who were clinic-radiologically suspected as case of Pott's spine were confirmed on FNAC as Pott's spine. Patients with Pott's spine should undergo radiologically guided FNAC as a routine part of their work up to rule out metastasis of the spine.

\section{Funding: Nil, Conflict of interest: Nil, Permission from IRB: Yes}

\section{References}

1. Aziz M, Afroz N, Kahkashan E, Ahmad I, Mansoor

T. Radiologically guided fine needle aspiration cytology of retroperitoneal and spinal lesions. J Cytol 2008;25:123-7

2. Francis IM, Das DK, Luthra UK, Sheikh Z, Sheikh M, Bashir M. Value of radiologically guided fine needle aspiration cytology (FNAC) in the diagnosis of spinal tuberculosis: a study of 29 cases. Cytopathology. 1999 Dec;10(6):390-401.

3. Bradley L. Coley, George S. Sharp, and Edward B. Ellis. Diagnosis of bone tumours by Aspiration. The American Journal of Surgery 1931;13(2):215-224

4. ELLIS F. Needle biopsy in the clinical diagnosis of tumours. Br J Surg. 1947 Jan;34(135):240-61.

5. TB Statistics India | Countrywide \& state statistics See more at: http://www.tbfacts.org/tb-statisticsindia/\#sthash.Jkbc7fx5.dpuf, accessed on $14^{\text {th }}$ Aug 2015.

6. MAZET R, COZEN L. The diagnostic value of vertebral body needle biopsy. Ann Surg. 1952 Feb;135(2):245-52.

7. Aggarwal R, Bandlish U, Mahajan S, Gupta N, Rambani R. Role of fine needle aspiration cytology in vertebral body lesions. Indian J Orthop 2002;36:46-8

8. Möller S, Kothe R, Wiesner L, Werner M, Rüther W, Delling G. Gluoroscopyguided transpedicular trocar biopsy of the spine-results, review, and technical notes. Acta Orthop Belg. 2001 Dec;67(5):488-99.

9. Mondal A. Cytological diagnosis of vertebral tuberculosis with fine-needle aspiration biopsy. J Bone Joint Surg am 1994 Feb;76(2):181-4.

10. Fyfe IS, Henry AP, Mulholland RC. Closed vertebral biopsy. J Bone Joint Surg Br. 1983 Mar;65(2):140-3.

\section{How to cite this article?}

Shukla A, Iyenger S, Jasmeet, Iyenger S.N. Role of radiologically guided FNAC in determining the lesions of spine. Int $J$ Med Res Rev 2015;3(8):810-814. doi: 10.17511/ijmrr.2015.i8.152. 